\title{
Does Famotidine Reduce the Risk of Progression to Severe Disease, Death, and Intubation for COVID-19 Patients? A Systemic Review and Meta-Analysis
}

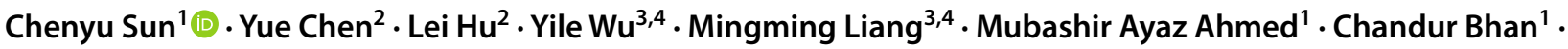 \\ Zhichun Guo ${ }^{5} \cdot$ Hongru Yang ${ }^{5} \cdot$ Yijing Zuo $^{5} \cdot$ Yue Yan $^{5} \cdot$ Qin Zhou $^{6}$
}

Received: 7 November 2020 / Accepted: 21 January 2021 / Published online: 24 February 2021

(c) The Author(s), under exclusive licence to Springer Science+Business Media, LLC part of Springer Nature 2021

\begin{abstract}
Background Famotidine was reported to potentially provide benefits to Coronavirus Disease 2019 (COVID-19) patients. However, it remains controversial whether it is effective in treating COVID-19.

Aims This study aimed to explore whether famotidine use is associated with reduced risk of the severity, death, and intubation for COVID-19 patients.

Methods This study was registered on International Prospective Register of Systematic Reviews (PROSPERO) (ID: CRD42020213536). A comprehensive search was performed to identify relevant studies up to October 2020. I-squared statistic and Q-test were utilized to assess the heterogeneity. Pooled risk ratios (RR) and 95\% confidence intervals (CI) were calculated through the random effects or fixed effects model according to the heterogeneity. Subgroup analyses, sensitivity analysis, and publication bias assessment were also conducted.

Results Five studies including 36,635 subjects were included. We found that famotidine use was associated with a statistically non-significant reduced risk of progression to severe disease, death, and intubation for Coronavirus Disease 2019 (COVID19) patients (pooled RR was $0.82,95 \% \mathrm{CI}=0.52-1.30, P=0.40$ ).

Conclusion Famotidine has no significant protective effect in reducing the risk of developing serious illness, death, and intubation for COVID-19 patients. More original studies are needed to further clarify whether it is associated with reduced risk of the severity, death, and intubation for COVID-19 patients.
\end{abstract}

Keywords Famotidine $\cdot$ COVID-19 $\cdot$ Meta-analysis

\section{Introduction}

A new type of $\beta$-coronavirus, severe acute respiratory syndrome coronavirus 2 (SARS-CoV-2), causing Coronavirus Disease 2019 (COVID-19) was first discovered in December 2019. The virus can be easily transmitted from person to person. Due to its strong pathogenicity [1-3], the virus can easily result in the spread of COVID-19 among population if limited or ineffective precautions are taken. Those infected with SARS-CoV-2 may present with fever, headache, and

An editorial commenting on this article is available at https://doi. org/10.1007/s10620-021-06875-w.

Chenyu Sun

drsunchenyu@yeah.net

Extended author information available on the last page of the article other mild symptoms, but in more severe cases, pneumonia, severe acute respiratory syndrome, and even death are also seen [4]. More than 34.8 million people were diagnosed with COVID-19 and 1,030,738 deaths were attributed to it as of October 4, 2020, making it a pandemic worldwide [5].

As a newly emerged infectious disease, evidence for treatment has been growing rapidly. Previous research has shown the potential role of famotidine [6], hydroxychloroquine [7], and remdesivir [8], in the treatment of COVID-19. Among them, famotidine was proposed to provide potential benefits, as a study identified famotidine could potentially inhibit the 3-chymotrypsin-like protease (3CLpro), which processes proteins that are essential for viral replication [9, 10]. Considering pathological histamine release and activation of dysregulated mast cells may be the factors leading to SARS-CoV2 infection, famotidine may act by antagonizing or anti-exciting histamine receptor $2[11,12]$. However, new 
emerging studies have now refuted the hypotheses and argue against the activity of famotidine as a direct-acting antiviral inhibitor of either the main or papain-like protease (PLpro) of SARS-CoV-2 [11, 13, 14].

It remains controversial whether famotidine is effective in treating COVID-19. On one hand, Freedberg et al. [6] found that famotidine could significantly reduce the risks of death or intubation. A lower level of certain serum markers was also observed. Mather et al. [15] agreed with their findings and shared similar opinions. On the other hand, Cheung et al. [16] found no association between famotidine use and COVID-19 severity. Hence, it is necessary to assess effects of famotidine on improving the outcome of those suffered COVID-19 by a meta-analysis.

\section{Methods}

This meta-analysis was reported in conformity to the Preferred Reporting Project declared by the Systematic Review and Meta-Analysis (PRISMA) [17]. And it was registered on International Prospective Register of Systematic Reviews (PROSPERO) (ID: CRD42020213536).

\section{Search Strategy}

A comprehensive search strategy was performed in Embase, PubMed, Cochrane Library, Web of Science, CNKI (China National Knowledge Infrastructure) China Biology Medicine (CBM), VIP (Chinese) database and Wanfang Data up to October 2020 to select relevant studies. The following terms were used: (histamine receptor antagonists OR histamine receptor blockers $\mathrm{OR} \mathrm{H} 2$ antagonist $\mathrm{OR} \mathrm{H} 2$ receptor antagonist OR H2RA OR H2 blockers OR ranitidine OR cimetidine OR nizatidine) AND (COVID-19 OR Novel coronavirus OR coronavirus disease OR SARS-CoV-2 OR Severe acute respiratory syndrome coronavirus 2 ). These words were replaced by Chinese phrases with the same meaning in Chinese databases.

\section{Inclusion Criteria and Exclusion Criteria}

Publications will be selected if they met the following inclusion criteria: (1) The research type was original studies; (2) the exposure was famotidine use without any other histamine receptor antagonists; (3) the subjects of the study were patients suffered from COVID-19; (4) the main outcome index is severe disease, death, and/or intubation rate; (5) hazard ratios (HRs) or relative risks (RRs) or odd ratios (ORs) were provided with its $95 \%$ confidence intervals (CIs) or enough data can be extracted to calculate the effect size; (6) second outcomes are the serum maker levels.
The following criteria were used to exclude studies:(1) The research was not human studies (such as reviews, metaanalyses, animal studies, or in vitro studies); (2) Cannot accurately determine the type of articles; (3) Unable to extract valid ending data and cannot calculate it; (4) Duplicate or studies reported the same data.

\section{Data Extraction and Quality Assessment}

Two reviewers (C. Sun and Y. Chen) looked through articles and extracted data independently. In case of discrepancies, consensus was reached by discussions and consulting with a third reviewer (Y. Wu). Information extracted contents include first author, geographic locations, sample size, exposure, dosage, outcomes, OR/HR/RR with its $95 \% \mathrm{CI}$, scores, adjustments. Newcastle-Ottawa Scale (NOS) was used to evaluate the quality of each study by two reviewers separately. A third person would be consulted to resolve the disparity when coming across disagreement. Any study that score of more than 7 stars was considered as a high-quality study, and those with a score between four and six stars were considered as moderatequality studies [18].

\section{Statistical Analysis}

The association between famotidine use and progression to severe disease, death, or intubation was analyzed through pooling the RRs and corresponding 95\% CIs. Considering the relative low incidence of severe cases, intubation, and death of COVID-19, HRs were viewed as RRs [19], and ORs are statistically transformed into RRs [20,21]. Then, the results were combined. Heterogeneity between the results of the included studies was analyzed by Q-test and evaluated in conjunction with the $\mathrm{I}^{2}$ statistic [22]. Fixed effects model was applied if the heterogeneity test showed non-significant results $\left(I^{2}<50 \%\right)$, otherwise a random effects model was applied $\left(I^{2}>50 \%\right)$ [23]. Pooled mean difference (MD) of ferritin level was analyzed by converting median to mean [24-28]. Sensitivity analysis was used to test the stability of results by excluding one study at a time to compare differences between the results after exclusion and the original results without exclusion [29]. Publication bias was visually assessed by a funnel plot and quantitatively assessed by Egger's test and Begg's test. [30, 31] All of the statistical analyses were performed through RevMan 5.3 and STATA 14.0. P values of less than 0.05 were taken to be statistically significant. 


\section{Results}

\section{Study Selection and Study Characteristics}

Through systematic search and retrieving records from other sources, 87 documents were initially obtained. After removing duplicate documents, 57 articles remained. By screening the title and the abstract, we filtered out 35 potentially relevant articles. After reading the full text carefully, five articles were included. Figure 1 shows the detailed process.

Five published articles $[6,15,16,32,33]$ with 36,635 participants were included. The NOS scores of the four studies were $\geq 6$ scores, which suggested moderate or high quality for the included studies. The basic characteristics of the included literature are shown in Table 1. The doses of famotidine of each study were also reported in Table 1 except for Chueng's study that did not specify the dose.

\section{Overall Meta-Analysis}

Heterogeneity was observed in the result $\left(I^{2}=76 \%\right.$, $P_{\text {heterogeneity }}=0.003$ ), and random effect model was applied. The pooled RR was $0.82(95 \% \mathrm{CI}=0.52-1.30, P=0.40)$, suggesting a non-significant effect of famotidine on reducing the risk of progression to severe disease, death, and intubation. (Fig. 2).

\section{Subgroup Analyses}

\section{Association Between Famotidine Use and Death and Intubation for COVID-19 Patients}

Further analysis of three studies regarding its effect on mortality and intubation $[6,15,32]$ did not find a statistically significant protective effect (RR:0.63, 95\% CI $=0.35-1.16$, $\left.P=0.140, I^{2}=84 \%, P_{\text {heterogeneity }}=0.002\right)$. Similarly, three studies investigating mortality only $[15,32,33]$ did not show a statistically significant protective effect (RR:0.90, $95 \% \mathrm{CI}=0.49-1.65, P=0.73, I^{2}=82 \%, P_{\text {heterogeneity }}=0.003$ ) (Table 2). We also analyzed studies with famotidine doses $\leq 40 \mathrm{mg}$, which happened to be the same three articles $[6,15,32]$ on mortality and intubation, showing the same result of pooled RR and 95\% CI.

\section{Association Between Famotidine Use and Serum Markers for COVID-19 Patients}

A lower median ferritin levels among famotidine users (708 ng/mL vs $846 \mathrm{ng} / \mathrm{mL}, P=0.030$ and $797.5 \mathrm{ng} / \mathrm{mL}$ vs. $964.0 \mathrm{ng} / \mathrm{mL}, P=0.076)$ were observed in two studies $[6$, 15]. A lower median CRP level $(9.4 \mathrm{mg} / \mathrm{mL}$ vs. $12.7 \mathrm{mg} /$ $\mathrm{dL}, P=0.002)$, median procalcitonin level $(0.16 \mathrm{ng} / \mathrm{mL}$ vs. $0.30 \mathrm{ng} / \mathrm{mL}, P=0.004)$, and median ESR level $(57.5 \mathrm{~mm} / \mathrm{h}$ vs. $68 \mathrm{~mm} / \mathrm{h}, P=0.190$ ) were also reported [15].

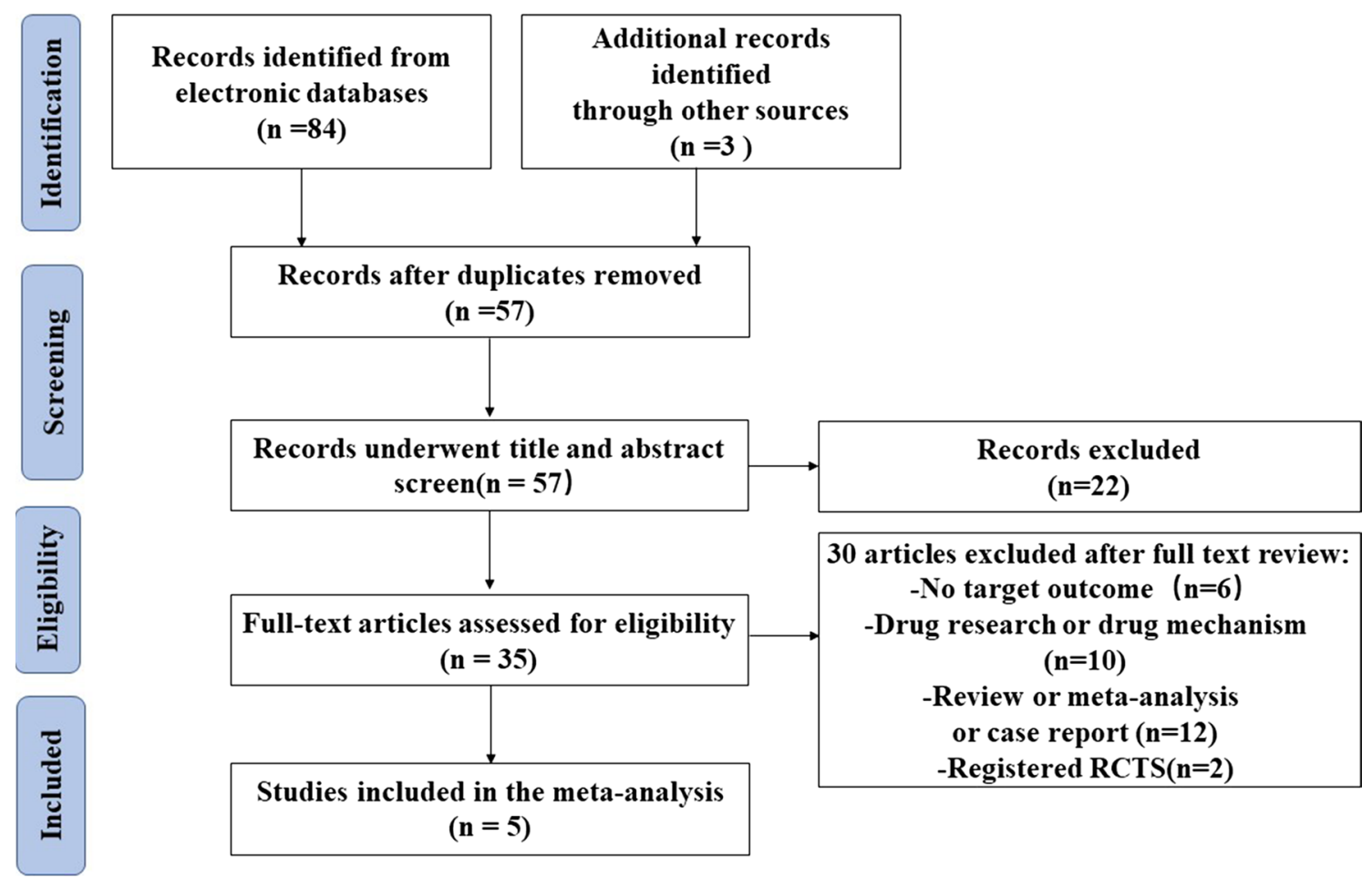

Fig. 1 PRISMA flowchart 


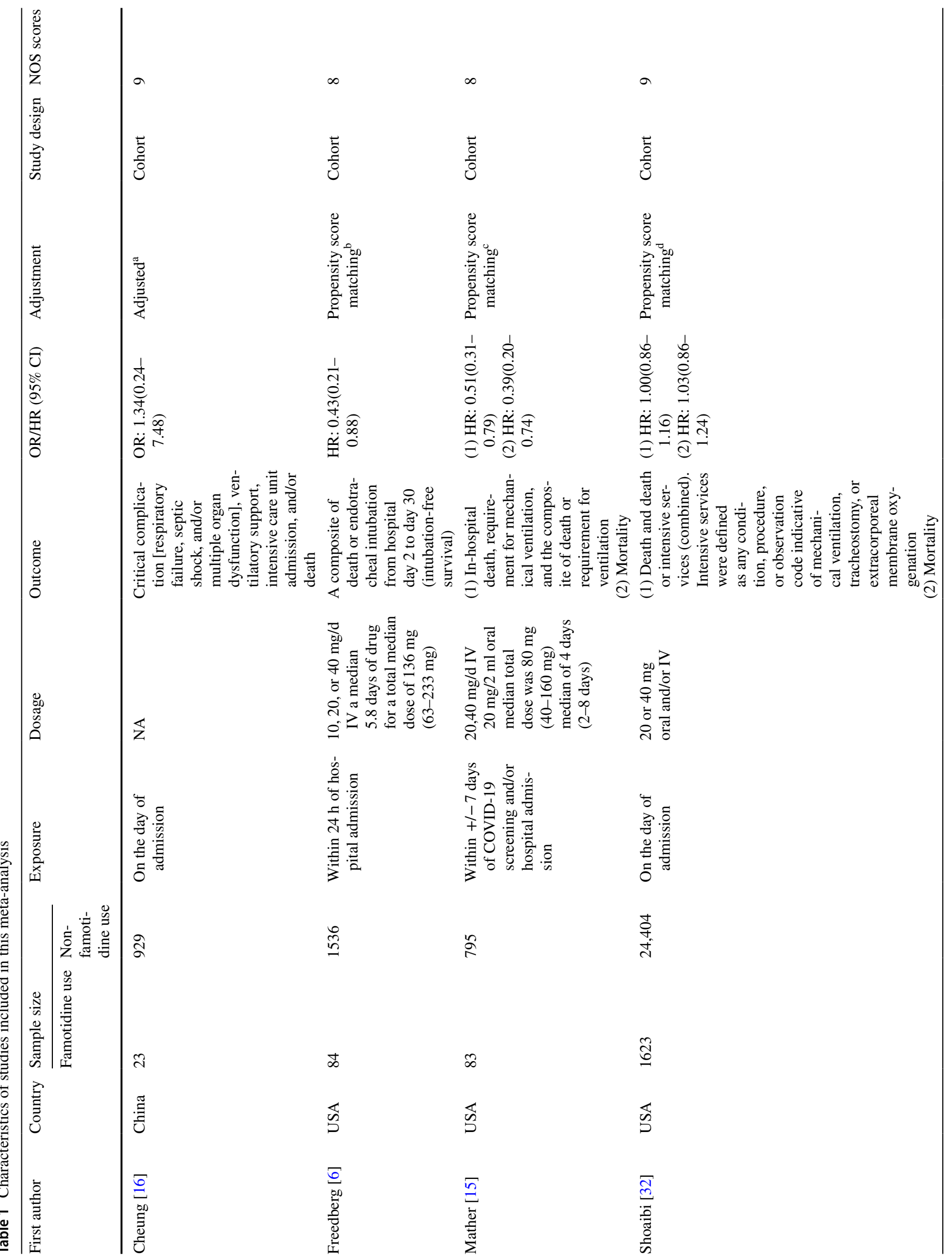




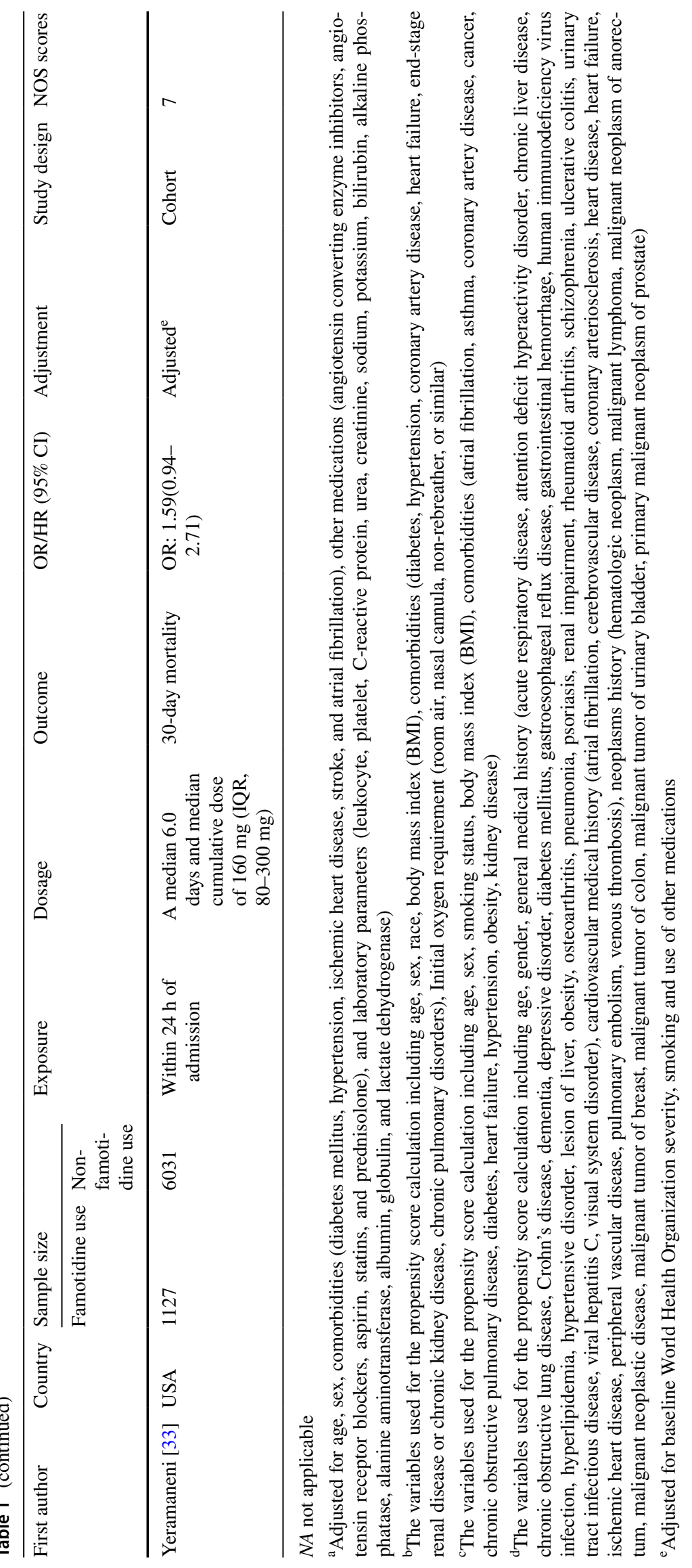


Pooled MD of ferritin level was analyzed by converting median to mean [24-28], and a reduced MD of -226.38 (95\% CI: $-333.95,-118.80, P<0.001 ; I^{2}=0 \%$, $P_{\text {heterogeneity }}=0.380$ ) was found.

\section{Sensitivity Analyses}

Sensitivity analysis was conducted by excluding each article and calculating the heterogeneity and effect size. The fluctuation of the pooled RRs was found to be between 0.68 and 0.97 with lower limit of $95 \%$ CI constantly remained less than 1 and upper limit of $95 \%$ CI constantly remained more than 1 , and $\mathrm{P}$ value constantly remained more than 0.05 , suggesting the stability of this meta-analysis. By changing the random effect model to fixed effect model, the overall result was not altered significantly ( $R R=0.94,95 \%$ CI: 0.83-1.08), further confirmed the stability.

\section{Publication Bias}

Funnel plot was shown to be symmetrical (Fig. 3). In addition, no publication bias was detected by Begg's test $(z=-0.24, P=1.000)$ and Egger's test $(t=-0.55$, $P=0.621)$.

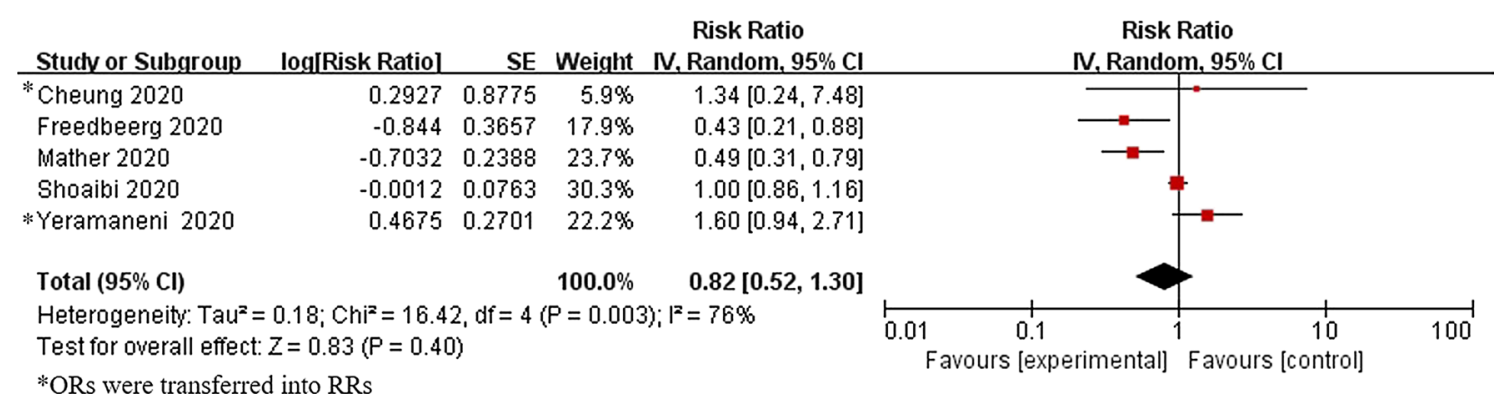

Fig. 2 Forrest plot: Association between famotidine and the risk of progression to severe disease, death, and intubation for COVID-19 patients

Table 2 Association between famotidine use and COVID-19 outcomes

Fig. 3 Funnel plot

\begin{tabular}{llllll}
\hline Analysis & $\begin{array}{l}\text { Number of } \\
\text { studies }\end{array}$ & OR (95\% CI) & $P$ & \multicolumn{2}{l}{ Heterogeneity } \\
\cline { 5 - 6 } & & & & $P_{\text {Heterogeneity }}$ & $I^{2}(\%)$ \\
\hline Severity, mortality and intubation & 5 & $0.82(0.52-1.30)$ & 0.400 & 0.003 & 76 \\
Mortality and intubation & 3 & $0.63(0.35-1.16)$ & 0.140 & 0.002 & 84 \\
Mortality only & 3 & $0.90(0.49-1.65)$ & 0.730 & 0.003 & 82 \\
\hline
\end{tabular}

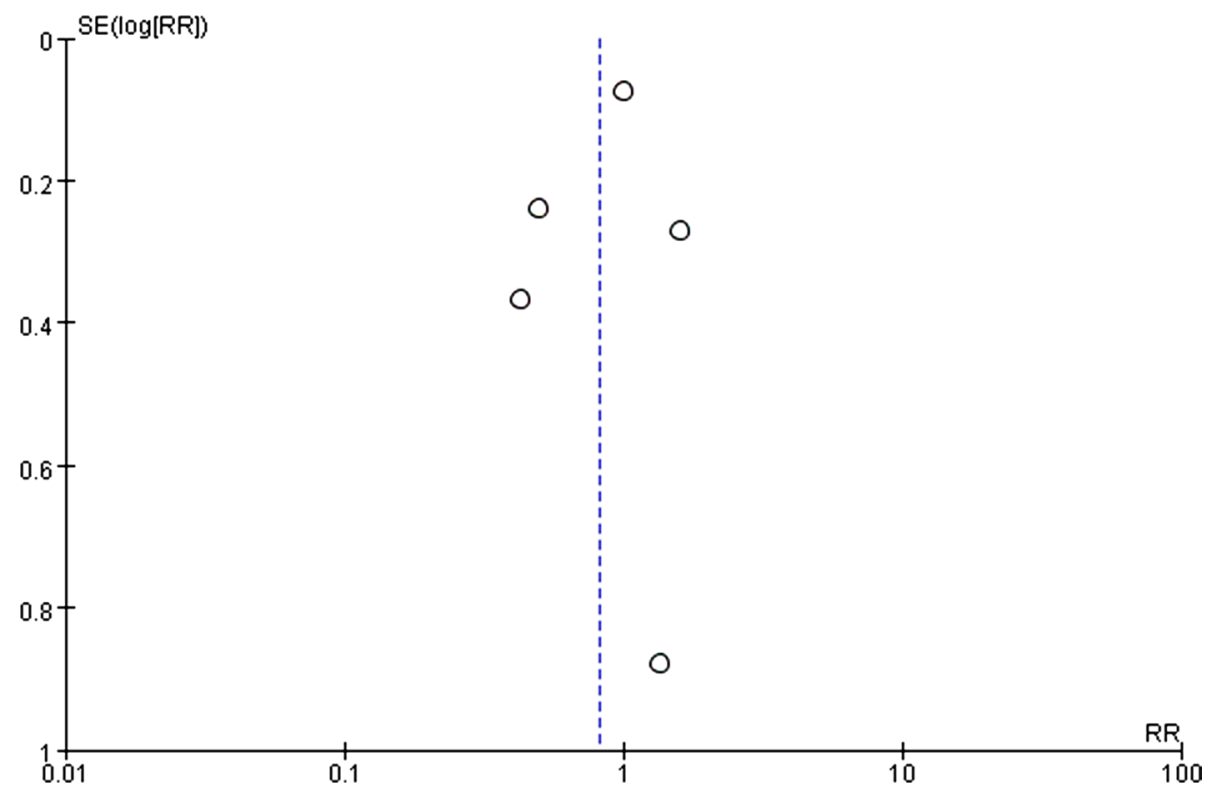




\section{Discussion}

In the overall pooled analysis, one study [16] reported severity (including critical complication, ventilatory support, ICU admission, and/or death), and three [6, 15, 32] reported death and intubation and one [33] reported death of COVID-19. Moreover, two randomized controlled trials (RCTs) were registered (ClinicalTrials.gov Identifier: NCT04370262 and NCT04545008), but no results have been reported yet. Consequently, famotidine was not associated with a reduced risk of progression to severe disease, intubation, and death of COVID-19. At the same time, subgroup analysis further confirmed the irrelevance between intubation and death and famotidine use. Moreover, three studies $[15,32]$ investigating mortality only did not find a protective effect, and one study [16] investigating severity only showed no association either. When sensitivity analysis was performed, the results of this meta-analysis did not change dramatically after excluding any of the included studies, suggesting the stability of this meta-analysis.

The combined results of the three studies $[6,15,32]$ on relatively low-dose famotidine were not statistically significant, suggesting that the standard over-the-counter (OTC) doses for treatment of gastroesophageal reflux disease (GERD) (20 mg PO per day to $40 \mathrm{mg}$ PO per day in split dose) of famotidine might be insufficient to yield significant clinical benefit in COVID-19 disease. However, this result should be interpreted with caution as only three studies were included in this subgroup. Freedberg's study concluded that famotidine reduced the risk of death and intubation, whereas the Shoaibi's article concluded that the assessment of both intubation and death, as well as death alone, was not statistically insignificant. In addition, only Freedberg's study and Yeramanei' study reported a cumulative dose, so it is difficult to assess the cumulative effect. It should also be noted that therapeutic efficacy of a pharmacological antagonist requires a steady-state concentration that substantially exceeds the half maximal inhibitory concentration (IC50) for its target. Famotidine has predicted steady-state concentration at different doses [11]. This might explain why higher than standard OTC doses of famotidine were reported to have potential benefits [34]. Nevertheless, published information indicating the effectiveness of higher doses of famotidine alone at treating COVID-19 in either inpatients or outpatients was very limited, especially no large cohort studies investigating the effectiveness of famotidine at COVID-19 treatment with doses higher than those used in gastroesophageal reflux disease (GERD) have been published yet. Therefore, how effective the higher dose of famotidine at treating COVID-19 remains speculative.

Although no decreased risk of severe illness, intubation, and mortality of COVID-19 was found among famotidine users, famotidine might still be potentially beneficial as lower serum markers were observed. A statically significantly lower median ferritin level [6], median CRP level, and median procalcitonin level [15] among famotidine users were also reported. For ferritin level, the pooled analysis of two studies $[6,15]$ also found a statistically significant lower level among famotidine users. Ferritin, CRP, procalcitonin, and other serum markers were thought to be potential prognosis predicators [35-37]. However, more investigation on these serum markers among COVID-19 patients on famotidine are needed to further elaborate this potential benefits.

According to the previous studies, famotidine may improve COVID-19 outcomes by several mechanisms. First, famotidine could potentially inhibit the 3-chymotrypsin-like protease (3CLpro), which processes proteins essential for viral replication $[9,10,15]$. Famotidine may also activate G-protein-coupled receptors (GPCRs) which was presumed to active immune cell mobilization, and result in vascular inflammation $[11,38]$. In contrast, Singh et al. found weak, nonspecific binding of famotidine to both PLpro and 3 chymotrypsin-like protease (3CLpro), which is the reverse of previous molecular docking studies [13]. And it was also recently reported that famotidine does not inhibit PLpro and Mpro nor does it inhibit SARS-CoV-2 infection [11, 14]. These controversial findings prompt the necessity of more investigation regarding the effect of famotidine on SARSCoV-2 infection. Second, mast cells activated by coronavirus were shown to produce histamine, prostaglandin D2 (PGD2), and leukotriene C4 (LTC4), inducing acute bronchoconstriction and lung inflammation [12]. Pneumocytes that are positive for $\mathrm{H} 1$ and $\mathrm{H} 2$ receptors could respond to local histamine release following mast cell degranulation [39]; therefore, famotidine and other H2RA may play a role in modulating the pulmonary pathological process. A recent cohort of 110 COVID-19 patients treated with famotidine and cetirizine by RB Hogan et al. exhibited that the combination of these two drugs can be very beneficial in terms of the incidence of death and progression of disease in hospitalized patients [40]. However, considering that our inclusion criteria require exposure to famotidine but not with other histamine receptor blockers, it was not included in the metaanalysis. Third, lung autopsy specimens have demonstrated a paucity of neutrophils and eosinophils in postmortem photomicrographs [41]. It is known that histamine 2 receptors could inhibit neutrophil effector functions such as oxygen release, chemotaxis induced by platelet-activating factor, and leukotriene biosynthesis [42-44], as well as inhibition of peroxidase release and chemotaxis by eosinophil [45, 46].

Several inherent limitations in this study should be mentioned. First, this meta-analysis included studies with relatively small sample size thus the results might be biased. Second, heterogeneity was noticed in overall analysis. The 
outcomes in the included studies are different, which may contribute to the heterogeneity. Third, in-hospital treatment and concomitant use of other medications use may also affect the COVID-19 outcome. But it is not adjusted for other in-hospital medications in the included studies. Fourth, pooled RR estimates had a bias because of the use of different RR indicators (HR or OR or RR) as the same effect measures. Fifth, two articles by Freedberg et al. and Mather et al. did not adjust for sufficient confounders. Last but not least, medians of ferritin levels were converted to mean for the pooled analysis, which might not be accurate for skewed distribution.

Despite the limitations, the advantages are as follows: First, our meta-analysis incorporated more articles and performed a more detailed analysis than the previous one [47]. Second, there was no publication bias. The sensitivity analysis found a robust result for the effects of famotidine, prompting our results to be credible.

In short, non-significant effect of famotidine on reducing the risk of progression to severe disease, death, and intubation. However, only limited number of original studies are available, more original studies are urgently needed to further clarify whether famotidine is associated with reduced risk of the severity, death, and intubation for COVID-19 patients.

Author's contribution CS designed the study, developed search strategy, performed literature search, collected the data, performed statistical analysis, and wrote the manuscript. YC performed literature search, developed search strategy, collected the data, statistical analysis, and wrote the manuscript. LH performed literature search and wrote the manuscript. YW developed search strategy, performed statistical analysis, revised the manuscript, and provided critical opinion. ML performed statistical analysis and interpreted the data. MAA and CB provided critical opinion and revised the manuscript. ZG, HY, YY, and YZ participated in writing and revised the manuscript. QZ provided critical opinion, participated in literature search, and revised the manuscript. All authors approved the final manuscript.

\section{Funding None.}

\section{Compliance with Ethical Standards}

Conflicts of interest The authors declare that they have no conflict of interest.

Protocol and registration The study protocol was prospectively registered in PROSPERO (http://www.crd.york.ac.uk/PROSPERO; registration number: CRD42020213536).

\section{References}

1. Zhu N, Zhang D, Wang W et al. China novel coronavirus investigating and research team. A novel coronavirus from patients with pneumonia in China, 2019. N Engl J Med. 2020;382:727-733
2. Wu Z, McGoogan JM. Characteristics of and Important lessons from the coronavirus disease 2019 (COVID-19) outbreak in china: summary of a report of 72,314 cases from the Chinese Center for disease control and prevention. JAMA 2020;323:1239-1242

3. Wu D, Wu T, Liu Q, Yang Z. The SARS-CoV-2 outbreak: what we know. Int J Infect Dis. 2020;94:44-48

4. Huang C, Wang Y, Li X et al. Clinical features of patients infected with 2019 novel coronavirus in Wuhan China. The Lancet 2020;395:497-506

5. WHO Coronavirus Disease (COVID-19) Dashboard. https://covid 19.who.int/. Accessed by 10.04.2020.

6. Freedberg DE, Conigliaro J, Wang TC et al. Famotidine use is associated with improved clinical outcomes in hospitalized COVID-19 patients: a propensity score matched retrospective cohort study. Gastroenterology 2020;159:1129-1131

7. Yang TH, Chou CY, Yang YF et al. Systematic review and metaanalysis of the effectiveness and safety of hydroxychloroquine in treating COVID-19 patients. J Chin Med Assoc. 2020. https://doi. org/10.1097/JCMA.0000000000000425.

8. Wilt TJ, Kaka AS, MacDonald R, et al. Remdesivir for adults with COVID-19: A living systematic review for an American college of physicians practice points. Ann Intern Med. 2020;M20-5752.

9. Wu C, Liu Y, Yang Y et al. Analysis of therapeutic targets for SARS-CoV-2 and discovery of potential drugs by computational methods. Acta Pharm Sin B 2020;10:766-788

10. Anand K, Ziebuhr J, Wadhwani P, Mesters JR, Hilgenfeld R. Coronavirus main proteinase (3CLpro) structure: basis for design of anti-SARS drugs. Science 2003;300:1763-1767

11. Malone RW, Tisdall P, Fremont-Smith P, et al. COVID-19: famotidine, histamine, mast cells, and mechanisms. Res $S q$ [Preprint]. 2020; 22:rs.3.rs-30934

12. Kritas SK, Ronconi G, Caraffa A, Gallenga CE, Ross R, Conti $P$. Mast cells contribute to coronavirus-induced inflammation: new anti-inflammatory strategy. J Biol Regul Homeost Agents 2020;34:9-14

13. Singh VP, El-Kurdi B, Rood C. What underlies the benefit of famotidine formulations used during COVID-19? Gastroenterology. 2020;S0016-5085L:35020-4.

14. Brandon J. Anson, Mackenzie E. Chapman, Emma K. Lendy et al. Broad-spectrum inhibition of coronavirus main and papain-like proteases by HCV drugs. Res Sq. 2020. https://doi.org/10.21203/ rs.3.rs-26344/v1

15. Mather JF, Seip RL, McKay RG. Impact of famotidine use on clinical outcomes of hospitalized patients with COVID-19. Am J Gastroenterol. 2020;115:1617-1623

16. Cheung KS, Hung IF, Leung WK. Association between famotidine use and COVID-19 severity in Hong Kong: a territory-wide study. Gastroenterology 2020;S0016-5085:34940-34944

17. Moher D, Liberati A, Tetzlaff J, Altman DG, PRISMA Group. Preferred reporting items for systematic reviews and meta-analyses: the PRISMA statement. Int J Surg. 2010;8:336-341

18. Stang A. Critical evaluation of the Newcastle-Ottawa scale for the assessment of the quality of nonrandomized studies in metaanalyses. Eur J Epidemiol. 2010;25:603-605

19. A glossary of EBM terms. https://bestpractice.bmj.com/info/us/ toolkit/ebm-tools/a-glossary-of-ebm-terms/ Accessed 28.11.2020.

20. Stare J, Maucort-Boulch D. Odds ratio, hazard ratio and relative risk. Metodoloski zvezki. 2016;13:59-67

21. Ronksley PE, Brien SE, Turner BJ et al. Association of alcohol consumption with selected cardiovascular disease outcomes: a systematic review and meta-analysis. BMJ 2011;342:479

22. Higgins JP, Thompson SG. Quantifying heterogeneity in a metaanalysis. Stat Med. 2002;21:1539-1558

23. DerSimonian R, Laird N. Meta-analysis in clinical trials. Control Clin Trials 1986;7:177-188 
24. Hozo SP, Djulbegovic B, Hozo I. Estimating the mean and variance from the median, range, and the size of a sample. BMC Med Res Methodol. 2005;20:5-13

25. Wan X, Wang W, Liu J, Tong T. Estimating the sample mean and standard deviation from the sample size, median, range and/or interquartile range. BMC Med Res Methodol. 2014;14:135

26. Bland M. Estimating mean and standard deviation from the sample size, three quartiles, minimum, and maximum. Int J Stats Med. 2015;4:57-64

27. Weir CJ, Butcher I, Assi V et al. Dealing with missing standard deviation and mean values in meta-analysis of continuous outcomes: a systematic review. BMC Med Res Methodol. 2018;18:25

28. Higgins JPT, Thomas J, Chandler J, et al. Cochrane Handbook for Systematic Reviews of Interventions version 6.1 (updated September 2020). Cochrane, 2020. www.training.cochrane.org/handbook.

29. Haidich AB. Meta-analysis in medical research. Hippokratia 2010;14:29-37

30. Begg CB, Mazumdar M. Operating characteristics of a rank correlation test for publication bias. Biometrics 1994;50:1088-1101

31. Egger M, Davey Smith G, Schneider M, Minder C. Bias in meta-analysis detected by a simple, graphical test. $B M J$ 1997;315:629-634

32. Shoaibi A, Fortin S, Weinstein R, Berlin J, Ryan P. Comparative effectiveness of famotidine in hospitalized COVID-19 patients. medRxiv. 2020. https://doi.org/10.1101/2020.09.23.20199463.

33. Yeramaneni S, Doshi P, Sands K, Cooper M, Kurbegov D, Fromell G. Famotidine use is not associated with 30-day mortality: a coarsened exact match study in 7158 hospitalized COVID-19 patients from a large healthcare system. Gastroenterology. 2020. https://doi.org/10.1053/j.gastro.2020.10.011.

34. Janowitz T, Gablenz E, Pattinson D et al. Famotidine use and quantitative symptom tracking for COVID-19 in non-hospitalised patients: a case series. Gut 2020;69:1592-1597

35. Li X, Ge P, Zhu J et al. Deep learning prediction of likelihood of ICU admission and mortality in COVID-19 patients using clinical variables. PeerJ 2020;8:e10337

36. Yan L, Zhang HT, Goncalves J et al. An interpretable mortality prediction model for COVID-19 patients. Nat Mach Intell. 2020;2:283-288

37. Mehta P, McAuley DF, Brown M et al. COVID-19: consider cytokine storm syndromes and immunosuppression. Lancet 2020;395:1033-1034
38. Birch CA, Molinar-Inglis O, Trejo J. Subcellular hot spots of GPCR signaling promote vascular inflammation. Curr Opin Endocr Metab Res 2021;16:37-42

39. Krystel-Whittemore M, Dileepan KN, Wood JG. Mast cell: a multi-functional master cell. Front Immunol. 2016;6:620

40. Hogan Ii RB, Hogan Iii RB, Cannon T et al. Dual-histamine receptor blockade with cetirizine-famotidine reduces pulmonary symptoms in COVID-19 patients. Pulm Pharmacol Ther. 2020;63:101942

41. Tian S, Hu W, Niu L, Liu H, Xu H, Xiao SY. Pulmonary pathology of early-phase $2019 \mathrm{~m}$ novel coronavirus (COVID-19) pneumonia in two patients with lung cancer. $J$ Thorac Oncol 2020;15:700-704

42. Burde R, Seifert R, Buschauer A, Schultz G. Histamine inhibits activation of human neutrophils and HL-60 leukemic cells viamH2-receptors. Naunyn Schmiedebergs Arch Pharmacol. 1989;340:671-678

43. Rabier M, Damon M, Chanez P et al. Inhibition by histamine of platelet-activating-factor-induced neutrophil chemotaxis in bronchial asthma. IntArch Allergy Appl Immunol. 1989;89:314-317

44. Flamand N, Plante H, Picard S, Laviolette M, Borgeat P. Histamine-induced inhibition of leukotriene biosynthesis in human neutrophils: Involvement of the $\mathrm{H} 2$ receptor and cAMP. $\mathrm{Br} J$ Pharmacol. 2004;141:552-561

45. Ezeamuzie CI, Philips E, Histamine H. (2) receptors mediate the inhibitory effect of histamine on human eosinophil degranulation. Br J Pharmacol. 2000;131:482-488

46. Wadee AA, Anderson R, Sher R. In vitro effects of histamine on eosinophil migration. Int Arch Allergy Appl Immunol. 1980;63:322-329

47. Sethia R, Prasad M, Jagannath S et al. Efficacy of famotidine for COVID-19: a systematic review and meta-analysis. medRxiv. 2020. https://doi.org/10.1101/2020.09.28.20203463.

Publisher's Note Springer Nature remains neutral with regard to jurisdictional claims in published maps and institutional affiliations.

\section{Authors and Affiliations}

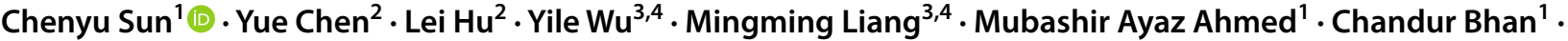 Zhichun Guo ${ }^{5} \cdot$ Hongru Yang $^{5} \cdot$ Yijing Zuo $^{5} \cdot{\text { Yue } \text { Yan }^{5} \cdot \text { Qin Zhou }}^{6}$}

1 Internal Medicine, AMITA Health Saint Joseph Hospital Chicago, 2900 N. Lake Shore Drive, Chicago, IL 60657, USA

2 Department of Clinical Medicine, School of the First Clinical Medicine, Anhui Medical University, No. 81 Meishan Road, Hefei 230032, Anhui, People's Republic of China

3 Department of Epidemiology and Health Statistics, School of Public, Health Anhui Medical University, No. 81 Meishan Road, Hefei 230032, Anhui, People's Republic of China
4 Center for Evidence-Based Practice, Anhui Medical University, No. 81 Meishan Road, Hefei 230032, Anhui, China

5 Massachusetts College of Pharmacy and Health Sciences, 179 Longwood Ave, Boston, MA 02115, USA

6 Radiation Oncology, Mayo Clinic, 200 First Street SW, Rochester, MN 55905, USA 